\title{
Phenomenological Study of the Experiences of Emergency Department Nurses in the Referral for and/or Administration of a Lethality Tool in a Rural Hospital
}

Michele I Bracken ${ }^{1}$ and Michelle Clifton ${ }^{2}$

${ }^{1}$ Department of Nursing, Salisbury University, Salisbury, Maryland, USA

${ }^{2}$ Atlantic General Hospital, Berlin, Maryland, USA

"Corresponding author: Michele I Bracken, Department of Nursing, Salisbury University, Salisbury, Maryland, USA, Tel: 410-543-6412; E-mail: mobracken@salisbury.edu

Rec date: Nov 25, 2014 Acc date: Dec 15, 2014 Pub date: Dec 20, 2014

Copyright: (c) 2014 Michele I Bracken. This is an open-access article distributed under the terms of the Creative Commons Attribution License, which permits unrestricted use, distribution, and reproduction in any medium, provided the original author and source are credited.

\begin{abstract}
Intimate partner violence is of national concern, resulting in an annual 4.9 million intimate partner physical and sexual assaults occurring in the United States. Due to resulting traumatic injuries, the emergency department is an ideal setting to assess for patients in abusive relationships. Because of this, it is critical that emergency department nurses involved in the screening process have perceptions and attitudes conducive to identification, care, and appropriate referral, to ensure the safety of these patients. Research has shown that nurses are not always effective in screening for intimate partner violence. There is a paucity of research on the attitudes and perceptions of these nurses that may provide a basis for this ineffective screening. The purpose of this research was to determine the attitudes and perceptions of emergency department nurses in a rural mid-eastern hospital regarding their experiences in referring for and/or administering an instrument that assess a patient's risk for being murdered by an abusive partner. Results of 9 interviews determined there were three emerging themes: 1) Worthwhile assessment tool 2) Barriers Encountered 3) Solutions to Barriers
\end{abstract}

Keywords: Phenomenological; Emergency Department; Nurses; Forensic

\section{Introduction}

Emergency department nurses are often the initial contact for interaction with an abused intimate partner. The attitude and perceptions of nurses toward patients reporting partner abuse has been found to be an important factor in subsequent interactions [1-4]. Patients in abusive relationships are reluctant to discuss their situation and depend on members of the health care team to bring up the subject, recognize their abuse and provide support and assistance [5-9]. Because attitude and perception is instrumental in the communication between the abused patient and the nurse, it is important to study the experiences of the emergency nurses who are at the forefront to determine if these experiences, or previous life experiences affect their referral for and/or administration of an instrument that measures how at risk that abused patient is for being murdered by their intimate partner.

Often victims of intimate partner abuse enter an emergency department for their injuries [10]. Unfortunately, the screening rates for the abuse in an emergency department are low, with a mere $13 \%$ of injured patients being screened for intimate partner violence [11]. It is of utmost importance that nurses in hospital emergency departments screen for intimate partner violence to decrease the $44 \%$ of women murdered by an intimate partner who had prior emergency department visits within just two years before their murder [12]. Previous studies cite barriers that impede the identification and subsequent referral of abused patients include concern for appearing intrusive by asking about abuse [13-15]. It is not enough to identify the barriers, it is essential to have an understanding behind these attitudes and perceptions in order to determine and implement effect strategies for change.

The purpose of this study was to make a contribution to what is known about the experiences of nurses who refer for and/or administer the Domestic Violence Lethality Screen for First Responders [16] to emergency department patients who identify partner abuse. This 11-question assessment determines a person's risk for being murdered by an abusive partner, and furthermore assists in identifying appropriate referrals geared toward safety. In order to increase detection of domestic violence, it is important for health care professionals to have an understanding of any attitudes and perceptions behind the barriers to the referral and/or administration process of a lethality tool in the emergency department of hospitals. Research which provides an understanding through the experiences of emergency department nurses may provide insight into possible development of interventions to decrease or eliminate these barriers [17]. This study explored the nursing staff's attitudes and perceptions of using this lethality tool with regard to the perceived challenges to patients and nurses.

\section{Methodology}

\section{Research questions}

This qualitative phenomenological study sought the perceptions of the nurses involved in referral and administration of a lethality tool. Information regarding the barriers to the implementation of this process implicated the need for an in-depth approach for better understanding of this phenomenon. The overall broad research question for this study was: "How do the experiences of emergency department nurses affect the referral for and/or the administration of a lethality tool?" 


\section{The following research questions were used to guide this} study:

- What are the perceived benefits to patients in referral for and administration of the Lethality Tool in an emergency department setting?

- What are the perceived benefits to nurses in referral for and administration of the lethality tool to emergency department patients?

- What are the perceived challenges/barriers to the process of referral for and administration of the lethality tool in the emergency department?

- How does referral for and administration of the lethality tool impact the work environment of the nurses?

- How does referral for and administration of the lethality tool impact the personal life of the nurses?

- How does referral for and administration of the lethality tool impact the nurses' perceptions of patients in abusive relationships?

- How does referral for and administration of the lethality tool impact the nurses' perceptions of patients who report abuse?

\section{Ethical considerations}

Intimate partner abuse is a sensitive research topic that has ethical implications. This section is provided to give an overview of the procedures that were undertaken to ensure informant well-being, and to meet ethical requirements. Approval for the conduction of this research was obtained from the Research Council and Ethics Committee of the rural hospital where the study took place as well as the Salisbury University Institutional Review Board before initiation of the study.

Individual interviews were conducted with 9 nurses who signed informed consents agreeing to be in the study as well as to have their interviews audio-taped. Potential risks discussed and included in the consent form included possible discomfort relating to past lived experiences related to referral for and/or administering the lethality tool, including caring for abused patients, and possibly having a personal history of abuse. To prevent the possible loss of confidentiality, no real names were included in the digital tape recordings, transcripts, or analyses. All materials were stored in secure cabinets and/or with secure passwords. The interviewer of all the participants was a women's health care nurse practitioner with expertise in qualitative research and intimate partner violence.

Possible benefits discussed and included in the consent form were to provide a voice for these participants in telling these experiences, an awareness of participation in a study that may identify barriers to the care of abused patients, participating in a research activity that may lead to Magnet accreditation for the organization as well as participating in a study to increase discipline knowledge and address the knowledge gap for nurses who refer for and/or administer a lethality tool in an emergency department nurses involved in this process, the overall possible benefit for such a study would be decreasing barriers to identifying, caring for and referring abused patients in an emergency room setting.

\section{History of the lethality assessment tool}

The Lethality Assessment Program (LAP) is the state of Maryland model [16]. The program was created in 2005 in conjunction with David Sargent (law enforcement trainer), the Maryland Network
Against Domestic Violence and adapted from Dr. Jacqueline Campbell's Danger Assessment with her assistance [18]. The tool was used by law enforcement officers to assess risk and connect victims of domestic violence for high risk as well as to connect them to services. In 2007, the emergency department forensic nurse examiners (FNE's) completed a 4-hour train-the-trainer education prepared and completed by David Sargent. These trained FNEs then expanded the scope of the screenings to include the Emergency Department charge nurses. There is an FNE on staff or on call 24/7 for the emergency department. The FNEs have an hour response time when on call. Should the FNE need the full 6minutes to respond, a delay in screening may occur. It was logical to expand the scope of screening to include the charge nurses allowing for immediate screening and intervention for the patient.

\section{Questions that trigger referral on the LAP tool}

The emergency department at this rural hospital uses a comprehensive tool for triage of the emergency department patient. The tool originally had a psychosocial screening question "Do you feel safe in your home?" among a few other vague questions. Two benefits emerged from the implementation of the LAP tool.

If a patient answered that they do not feel safe in their home, the nurse now had a resource to provide intervention for the patient.

The psychosocial questions were updated to be better:

Psychosocial

- Evidence of Abuse/Neglect/Self Harm Yes* No

- Do you feel safe in your home? Why not? Yes $\mathrm{No}^{\star}$

- Is there anyone you are afraid of? Yes ${ }^{\star}$ No

- Is anyone hurting you? Yes* No

- Family needs assistance to comply with plan of care? Yes ${ }^{\star}$ No

- Do you have any intentions or thoughts of harming yourself? Yes* No

- Need for psychosocial/spiritual or cultural referral? Yes ${ }^{\star}$ No

- Self-harm Assessment Trigger (Yes to 1 \& 6) Yes No

- Lethality Assessment Trigger (no to 2, yes to 3,4 Yes No

\section{Results and data interpretation}

After interviews were analyzed, themes emerged. Data analysis was ongoing and adherence to protocol was followed as to maintain the focus on answering the research questions according to the phenomenological approach. Validity was addressed by way of conveying an understanding by the researcher of basic philosophical tenets of phenomenology as well as ensuring that the essence of the phenomenon was captured by the description and interpretation of the researcher. The use of reflection was utilized to come to an understanding of the phenomenon and interpreted by the researcher. Memos as well as field notes documenting the process were kept in a journal that established an audit trail. Themes that emerged from the data were: 1) Worthwhile Tool, 2) Barriers Encountered, and 3) Solutions to Barriers.

Participants in the study felt that the lethality tool is worthwhile. One participant responded, "It helps by showing that someone else cares and they don't have to live like this." Another respondent was in 
agreement, adding, "It is a good thing to give them resources." Several respondents felt the length of the tool was an advantage, "It is short, sweet...gets right to the point."

The second theme that emerged from the study was that there were barriers encountered with the administration of and/or referral of the lethality tool. Most of the participants felt a challenge they encountered was that there was a difficulty administering the tool in a private area, saying, "Not private...difficult to get the abuser away." Many participants were frustrated that once they administered the tool and connected them by referral, the victims' outcomes were unknown to them. One participant expressed this by saying, "I haven't seen the final effects...don't know what happened to her." Another barrier dealt with the exhaustion of dealing with the administration of the tool. One participant said, "It can be emotionally and mentally taxing...takes time...don't want to rush...don't want to take it home." By "taking it home", the participant meant that she did not want to carry the emotional weight of the assessing process back to her family. All of the participants offered solutions to these barriers they encountered. Many said that they "Could use debriefing", especially after particularly draining encounters. There were, in fact, protocols set up for such debriefing at their workplace, but some of the participants were unaware these existed. Most of the participants felt they "should have annual training with role playing" to help them stay fresh with their administration of the tool as well as their experiences with the victims and their abusers. It was felt by all participants that they "could use more education of what the tool indicates". They felt this could be incorporated into the annual trainings.

\section{Discussion}

Women who experience intimate partner violence are often seen in emergency departments $[19,20]$. The themes that emerged from this study predominantly dealt with communication. Results indicated that participants felt the LAP was a worthwhile tool to administer to victims of intimate partner violence to determine their risk for danger and to initiate support and referral. Much of the research related to domestic violence screening has been about communication from the perspective of the victims as well as their health care providers regarding the difficulties in disclosure [5,7,9,21-25].

The participants in this study felt that it was important for their abuse patients to know someone cared and provided them with resources. Research has found that use of caring allowed their patients to feel understood and visible to the health care providers [24,26-31]. Providing services such as resources was found to be important to abuse victims [32-35]. Providing a respectful dialogue has been shown in research to be of great importance to victims of abuse [7]. There is much evidence to support that the communication between health care provider and abuse victim can effect change in their lives if done with compassion and without judgment $[7,9]$. Trust has been shown to be an important element during communication between the healthcare provider and the abused victim $[30,33]$.

In this study, the emergency department nurses suggested solutions to some of the barriers inherent in administering the LAP. One solution posed by the participants in this study was a desire to have annual workshops that would include role play to aid in their confidence in administration of the lethality tool. Role play has been associated with increased communication skill in screening for domestic violence [36].

\section{Conclusions}

Any emergency department can be busy and the added task of administering the lethality tool may be felt to be a burden in an already environment with time constraints. Although limited time has been reported as a barrier in studies [37-39], it has also been shown that substantial additional time is not added by this process [40]. Time was a theme that emerged in this study, although while some participants felt the tool was concise, others expressed concern that the process took time away from their other patients.

Perhaps facilities should take stronger measures to ensure that their employees are aware of the debriefing policies that could provide them with the relief that may address and decrease the emotional exhaustion expressed by the participants in this study. It was also brought out in this study that the emergency nurses were disappointed and frustrated that they were not told about the outcome of their assessment and referrals. This may be an area that facilities could address to keep their nurses in the loop of the care provided to their patients. Future research might endeavor to determine how an annual workshop involving education and role-play may improve their communication skills in dealing with victims of intimate partner violence. Providing a workshop that involves role play may assist the healthcare provider to identify ways in which to separate the abuser from the victim in order to be able to privately administer the lethality assessment tool as well as practicing with the assessment to assure more time efficiency while not compromising an attitude of caring. It is of the utmost importance to show concern, advocacy and collaboration during any LAP assessment [41].

\section{References}

1. Gutmanis I, Beynon C, Tutty L, Wathen C, MacMillan H (2007) Factors influencing identification of and response to intimate partner violence: a survey of physicians and nurses. Biomedical Medical Central Public Health $7: 12$.

2. Häggblom AM, Hallberg LR, Möller AR (2005) Nurses' attitudes and practices towards abused women. Nursing and Health Sciences 7: 235-242.

3. Häggblom AM, Möller AR (2006) A life-saving mission: nurses' willingness to encounter with intimate partner abuse. Qualitative Health Research 16: 1075-1090.

4. Woodtli MA (2001) Nurses attitudes toward survivors and perpetrators of domestic violence. Journal of holistic nursing: official journal of the American Holistic Nurses' Association 19: 340-359.

5. Bracken M (2008) Leaving an abusive relationship: A hermeneutic phenomenological study of lifetime experiences of women who have left abusive relationships. Dissertation, University of Maryland, Baltimore.

6. Bracken M, Messing JT, Campbell JC, LaFlair L, Kub J (2010) Intimate partner violence and abuse among female nurses and nursing personnel: Prevalence \& risk factors. Mental Health Nursing, Special Issue on Violence Exposure on Children, Adolescents and Women: The Lived Experience 31: 1137-1148.

7. Chang JC, Decker M, Moracco KE, Martin SL, Petersen R et al., (2003) What happens when health care providers ask about intimate partner violence? A description of consequences from the perspectives of female survivors. J Am Med Womens Assoc 58: 76-81.

8. Edwards M (2005) Raising the subject of domestic violence. Practice Nurse 29: 26-30.

9. Yam M (2000) Seen but not heard: battered women's perceptions of the ED experience. J Emerg Nurs 26: 464-470.

10. Kothari CL, Rhodes KV (2006) Missed opportunities: emergency department visits by police-identified victims of intimate partner violence, Annals of Emergency Medicine 47: 190-199. 
Citation: Michele IB, Michelle C (2015) Phenomenological Study of the Experiences of Emergency Department Nurses in the Referral for and/or Administration of a Lethality Tool in a Rural Hospital. J Forensic Res 6: 1000265. doi:10.4172/2157-7145.1000265

Page 4 of 4

11. Garcia-Moreno C (2002) Dilemmas and opportunities for an appropriate health-service response to violence against women. Lancet 359: 1509-1514.

12. Crandall ML, Nathens AB, Kernic MA (2004) Predicting future injury among women in abusive relationships. Journal of Trauma 56: 906-912.

13. Waalen J Goodwin, Spitz MM, Petersen AM, Saltzman RLE (2000) Screening for intimate partner violence by healthcare providers. Barriers and interventions. Am J Prev Med 19: 230-237.

14. Taft A Broom and Legge DH (2004) D General practitioner management of intimate partner abuse and the whole: qualitative study. British Medical Journal 32: 618-622.

15. Bair-Merritt M, Giardino AP, Turner M, Ganetsky M, Christian CW (2004) Pediatric residency training on domestic violence: A national survey. Ambulatory Pediatrics 4: 24-27.

16. How is the LAP Making an Impact in Maryland? LAP: Maryland. Retrieved September 7, 2013

17. Cucu-Oanecea $O$ (2010) The reuse of qualitative data in the social sciences. Archiving and analysis. Sociologia 141-160.

18. Abbott J Johnson (2013) What is the Danger Assessment? (n.d.).

19. R Koziol-McLain, J Lowenstein (1995) SR. Domestic violence against women. Incidence and prevalence in an emergency department population. JAMA 273: 1763-1767.

20. Muelleman RL, Lenaghan PA, Pakieser RA (1998) Nonbattering presentations to the ED of women in physically abusive relationships. Am J Emerg Med 16: 128-131.

21. Rhodes KV, Frankel RM, Levinthal N, Prenoveau E, Bailey J et al., (2007) "You're not a victim of domestic violence, are you?". Provider patient communication about domestic violence. Ann Intern Med 147: 620-627.

22. Gilbert B, Johnston K, Caspers N, Bleecker T, Woods A et al., (1996) Experience of battered women in health care settings: a qualitative study. Women and Health 24: 1-7.

23. Gremillion DH, Evins G (1994) Why don't doctors identify and refer victims of domestic violence? N C Med J;55: 428-432.

24. McCauley J, Yurk RA, Jenckes MW, Ford DE (1998) Inside "Pandora's box": abused women's experiences with clinicians and health services. J Gen Intern Med 13: 549-555.

25. Rodriguez MA, Quiroga SS, Bauer HM (1996) Breaking the silence. Battered women's perspectives on medical care. Arch Fam Med 5: 153-158.

26. Suchman AL, Markakis K, Beckman HB, Frankel R (1997) A model of empathic communication in the medical interview. JAMA 277: 678-682.
27. Levinson W, Gorawara-Bhat R, Lamb J (2000) A study of patient clues and physician responses in primary care and surgical settings. JAMA 284 1021-1027.

28. Epstein RM, Morse DS, Frankel RM, Frarey L, Anderson K et al., (1998) Awkward moments in patient-physican communication about HIV risk. Ann Intern Med 128: 435-442.

29. Branch and Malik (1993) Using 'Windows of Opportunities' in Brief Interviews to Understand Patients' Concerns 269:1667-1668

30. Gerbert B, Caspers N, Bronstone A, Moe J, Abercrombie P (1999) A qualitative analysis of how physicians with expertise in domestic violence approach the identification of victims. Ann Intern Med 131: 578-84.

31. Limandri B (1989) Disclosure of stigmatizing conditions: the discloser's perspective. Archives of Psychiatric Nursing 3: 69-78.

32. Nicolaidis C (2002) The Voices of survivors documentary: using patient narrative to educate physicians about domestic violence. J Gen Intern Med 17:117-124.

33. Gerbert B, Abercrombie P, Caspers N, Love C, Bronstone A (1999) How health care professionals help battered women: The survivor's perspective. Women and Health 29: 115-135.

34. Dienemann J, Glass N, Hyman R (2005) Survivor preferences for response to IPV disclosure. Clin Nurs Res 14: 215-237.

35. Rodriguez MA, Sheldon WR, Bauer HM, Perez-Stable EJ (2001) The factors associated with disclosure of intimate partner abuse to clinicians. J Fam Pract 50: 338-344.

36. Edwardsen EA, Morse DS, Frankel RM (2006) Structured practice opportunities with a mnemonic affect medical student interviewing skills for intimate partner violence. Teach Learn Med 18: 62-68.

37. Brown JB, Lent b, Sas G (1993) Identifying and treating wife abuse. J Fam Prac 36: 185-191.

38. Davies J, Harris M, Roberts G et al., (1996) Community health workers' response to violence against women. Austr NZ J Ment Health Nurs 5: 20-32.

39. Sugg NK and Inui T (1992) Primary care physicians' response to domestic violence: opening Pandora's box. JAMA 267: 65-68.

40. Steward M, Brown J, Weston W (1989) Patient-centered interviewing: five provocative questions. Canadian Family Physician 35: 159-161.

41. Campbell J (2002) Health consequences of intimate partner violence. The Lancet 359: 1331-1336. 